\title{
IMPEDANCE SPECTROSCOPY AND AC CONDUCTIVITY STUDIES OF FERROELECTRIC $\left(\mathrm{K}_{0.5} \mathrm{Na}_{0.5}\right) \mathrm{NbO}_{3}$ CERAMICS
}

\author{
P. PALEI* and P. KUMAR ${ }^{\dagger}$ \\ National Institute of Technology \\ Rourkela 769008, India \\ *prakash.palei@gmail.com \\ †pawankumar@nitrkl.ac.in
}

Received 26 April 2011

\begin{abstract}
Lead free $\left(\mathrm{K}_{0.5} \mathrm{Na}_{0.5}\right) \mathrm{NbO}_{3}(\mathrm{KNN})$ ceramics were prepared by conventional solid state reaction route. For single perovskite phase formation, calcination temperature was optimized at $850^{\circ} \mathrm{C}$ for $6 \mathrm{~h}$, whereas for dense morphology the sintering of the ceramic was carried out at $1120^{\circ} \mathrm{C}$ for $4 \mathrm{~h}$. $\mathrm{X}$-ray diffraction XRD analysis confirmed the formation of single phase with orthorhombic structure at room temperature. Impedance analysis and AC conductivity studies of the KNN sample was carried out in the temperature range of $703-773 \mathrm{~K}$. Impedance study showed the increase in conducting behavior at higher temperature. The temperature dependence of AC conductivity indicated that the conduction process is due to doubly ionized oxygen vacancies in the higher temperature region.
\end{abstract}

Keywords: Perovskite; sintering; AC conductivity; impedance; oxygen vacancies.

\section{Introduction}

In recent years, there is an increasing demand for the use of environmental friendly lead-free ceramics in piezoelectric actuators, sensors and transducers applications. ${ }^{1-3}$ One such important ceramic is potassium sodium niobate, $\left(\mathrm{K}_{0.5} \mathrm{Na}_{0.5}\right) \mathrm{NbO}_{3} /(\mathrm{KNN})$, which has attracted a lot of attention because of having high Curie temperature $\left(T_{\mathrm{C}}\right)$, good electrical properties and environmental friendly nature. ${ }^{4-9}$ KNN is a solid solution of potassium niobate (KN), a ferroelectric system, and sodium niobate (NN), an anti-ferroelectric system. This KNN system possesses a morphotropic phase boundary (MPB) similar to PZT (52/48) system when the $\mathrm{Na} / \mathrm{K}$ ratio is $\sim 50 / 50$. The excellent properties of KNN ceramics are considered due to the presence of a MPB similar to PZT ceramics. Inspite of having promising properties, there are some problems associated with the KNN ceramics during processing. One major problem is the volatization of alkali elements at higher sintering temperature, as a result of which oxygen vacancies are created which increases the electronic conductivity of the ceramics. ${ }^{10,11}$ The electrical properties of KNN-based ceramics at high temperatures (above $T_{\mathrm{C}}$ ) have been rarely studied due to its high dielectric $\operatorname{loss}(\tan \delta)$. This higher values of $\tan \delta$ at high temperature is reported due to the net effect of space charge and defects/carrier contributions. ${ }^{12}$ However, by the high temperature investigations, it is possible to separate out these contributions. Moreover, in all ferroelectrics, the study of electrical conductivity is very important since the associated physical

\footnotetext{
† Corresponding author.
} 
properties like piezoelectricity, ferrroelectricity and also strategy for poling are dependent on the order and nature of conductivity in these materials. Impedance spectroscopy is a powerful tool for the study of the electrical properties of ionic, electronic or mixed conductor ceramics. It has already been applied successfully in the investigation of various other ferroelectric materials. ${ }^{13-15}$

In view of the increased interest in KNN ceramics, this work has been dedicated to a detailed study of electrical behavior using AC conductivity and impedance spectroscopy techniques.

\section{Experimental Procedure}

KNN ceramics were synthesized by conventional solid state reaction route (CSSR). Sodium carbonate $\left(\mathrm{Na}_{2} \mathrm{CO}_{3}, \quad 99.9 \%\right.$ purity), potassium carbonate $\left(\mathrm{K}_{2} \mathrm{CO}_{3}, 99.9 \%\right.$ purity) and niobium pentoxide $\left(\mathrm{Nb}_{2} \mathrm{O}_{5}, 99.9 \%\right.$ purity $)$ were used as starting precursors. Stoichiometric weights of all the powders were mixed and ball milled with acetone for $8 \mathrm{~h}$, using zirconia balls as the grinding media. The calcination was carried out at $850^{\circ} \mathrm{C}$ for $6 \mathrm{~h}$ and single perovskite phase formation was confirmed by X-ray diffraction (XRD) technique. The calcined powder was mixed thoroughly with $2 \mathrm{wt} \%$ polyvinyl alcohol (PVA) binder solution and then pressed into disks of diameter $\sim 10 \mathrm{~mm}$ and a thickness of $\sim 1.5 \mathrm{~mm}$ under $60 \mathrm{MPa}$ pressure. The binder burnout was completed by firing the sample at $600^{\circ} \mathrm{C} / 2 \mathrm{~h}$. The sintering of the samples was carried out at $1120^{\circ} \mathrm{C}$ for $4 \mathrm{~h}$ in air with a heating rate of $5^{\circ} \mathrm{C} / \mathrm{min}$. In order to examine the phases present in the system, XRD analysis of the sintered KNN ceramics were performed on a PW 3020 Philips diffractometer using $\mathrm{Cu} \mathrm{K} \alpha(\lambda=0.15405 \mathrm{~nm})$ radiation. The experimental densities $\left(d_{\mathrm{ex}}\right)$ of the samples were calculated by the Archimedes method. Silver paste was applied on both sides of the samples for the electrical measurements. Dielectric constant $\left(\varepsilon_{\mathrm{r}}\right)$ and dielectric loss $(\tan \delta)$ were measured as a function of frequency and temperature using an impedance analyzer (HP 4192 LF).

\section{Results and Discussion}

The XRD patterns of the KNN ceramics calcined at $850^{\circ} \mathrm{C} / 6 \mathrm{~h}$ and sintered at $1120^{\circ} \mathrm{C} / 4 \mathrm{~h}$ are shown in Fig. 1. XRD peaks confirm the formation of single perovskite phase without any trace of secondary

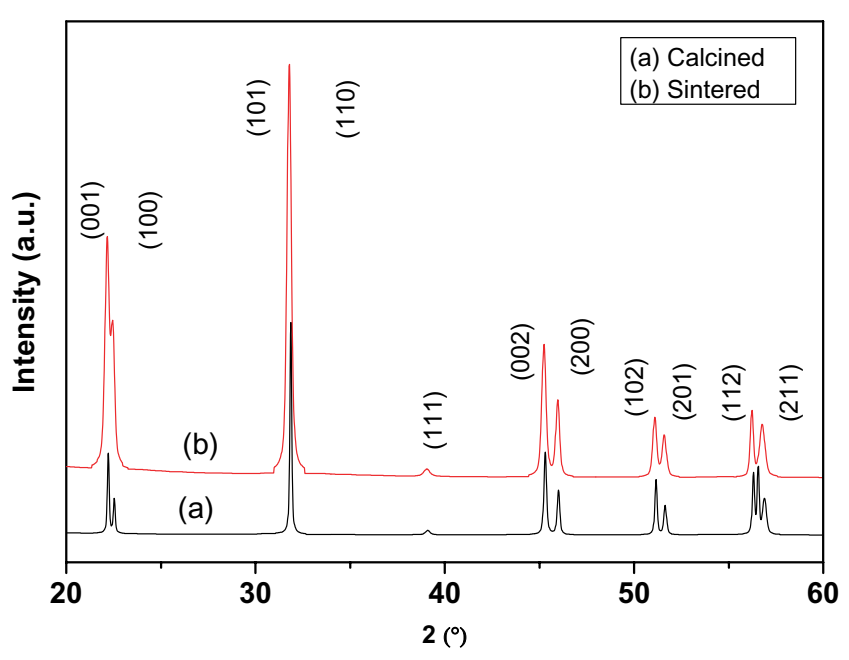

Fig. 1. XRD patterns of KNN samples (a) calcined at $850^{\circ} \mathrm{C} /$ $6 \mathrm{~h}$ and (b) sintered at $1120^{\circ} \mathrm{C} / 4 \mathrm{~h}$.

phase peaks. The XRD peaks are found to be sharp and distinct indicating good homogeneity and crystallinity of the sintered KNN samples. ${ }^{16}$ The diffraction lines of the sintered KNN ceramics were indexed in different crystal systems and unit cell configurations using a computer program package "Powdmult." Standard deviations (SD), $\sum \Delta d=$ $\left(d_{\text {obs }}-d_{\text {cal }}\right)$, where " $d$ " is inter-plane spacing, is found to be minimum for orthorhombic structure. The lattice parameters and unit cell volume are found to be $a=3.9388 \AA, b=3.9953 \AA, c=4.0280 \AA$ and $V=63.39 \AA^{3}$, whereas the X-ray density $\left(d_{x}\right)$ of the sample was found to be $\sim 4.5056 \mathrm{~g} / \mathrm{cc}$. The X-ray density of the sample was calculated by using the formula

$$
d_{x}=\frac{\left(\sum A / N\right)}{V} n,
$$

where $d_{x}$ is the X-ray density, $\sum A$ is the sum of the atomic weights of all the atoms in the unit cell, $N$ is the Avogadro's number, $V$ is the volume of the unit cell and $n$ is the number of atoms per unit cell of the crystal structure.

Figure 2 shows the temperature dependence of $\varepsilon_{\mathrm{r}}$ at different frequencies of KNN samples sintered at $1120^{\circ} \mathrm{C}$. It can be seen that there exists two sharp transition peaks. The first peak $\sim 198^{\circ} \mathrm{C}$ is related with the polymorphic phase transition (orthorhombic to tetragonal) and the second one located at $\sim 425^{\circ} \mathrm{C}$ is the ferroelectric to paraelectric phase transition temperature (Curie temperature) of the KNN ceramics. The value of $\varepsilon_{\mathrm{r}}$ increases with the increase in temperature. In the high temperature 


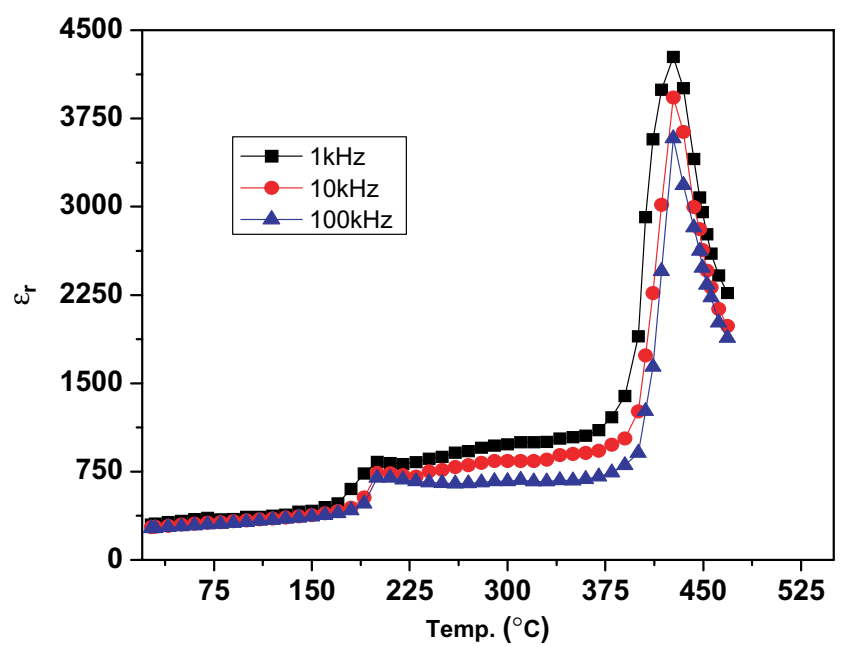

Fig. 2. Temperature variation of dielectric constant $\left(\varepsilon_{\mathrm{r}}\right)$.

region, higher value of $\varepsilon_{r}$ may be due to the contributions from space charge polarization, which comes from mobility of ions and imperfections in the material. ${ }^{17}$ These combined effects produce a sharp increase in the value of $\varepsilon_{\mathrm{r}}$ with the increase in temperature.

In order to understand the dielectric relaxation phenomena, Cole-Cole study has been used to analyze the impedance data in the temperature range from 703 to $773 \mathrm{~K}$. Figure 3 shows the Cole-Cole plots of KNN ceramics above Curie temperature in the frequency range $(100 \mathrm{~Hz}-1 \mathrm{MHz})$. At lower temperature $(<703 \mathrm{~K})$ there is a linear response in $Z^{\prime \prime}$. This trend indicates the good insulating behavior in the sample. As the temperature increases above $713 \mathrm{~K}$, the linear response gradually changes to semicircle in nature. This shows the conducting

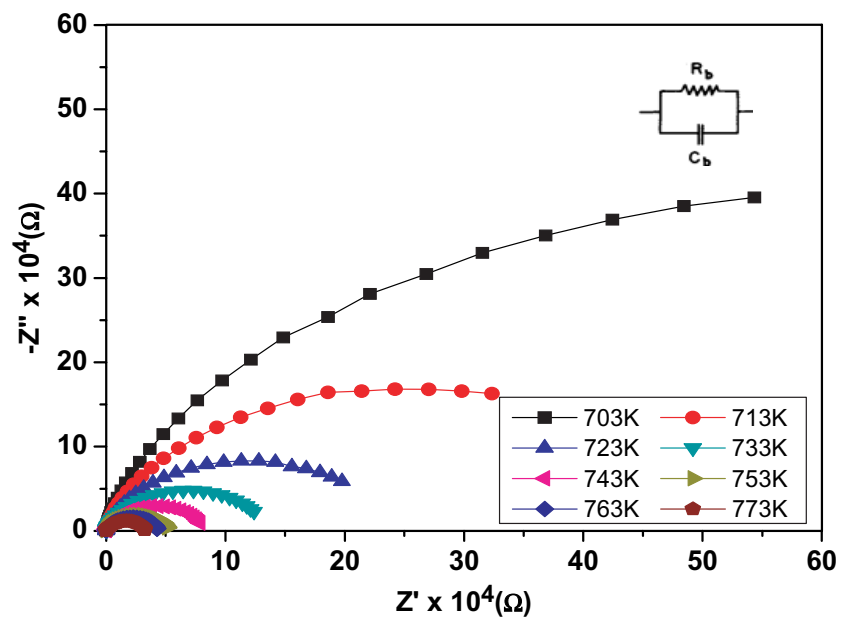

Fig. 3. (Color online) Complex impedance plots for KNN ceramics at different temperatures. behavior in the sample increases at higher temperature. A single semicircular arc has been observed in a wide temperature range. This indicates that the electrical properties of the material arise mainly due to the bulk effects. It also shows that as the temperature increases intercept point on the real axis shifts toward the origin indicating the decrease in the resistive property of the material. This gives the bulk resistance $\left(R_{\mathrm{b}}\right)$ of the material. This type of electrical behavior can be explained in terms of an equivalent circuit comprising of a parallel combination of bulk resistance $\left(R_{\mathrm{b}}\right)$ and bulk capacitance $\left(C_{\mathrm{b}}\right)$ (inset Fig. 3). The value of $R_{\mathrm{b}}$ and $C_{\mathrm{b}}$ is shown in Table 1 . Figure $4(\mathrm{a})$ shows real parts of impedance $\left(Z^{\prime}\right)$ as a function of frequency at various temperatures. It is evident that $Z^{\prime}$ decreases with increase in both frequency as well as temperature. The $Z^{\prime}$ values for all temperature shows dispersion at low frequency region which may be due to the presence of thermally assisted electric or ionic relaxation process. The $Z^{\prime}$ values for all the temperatures merge above $10 \mathrm{kHz}$ frequency, which indicates the presence of space charge polarization in the sample. ${ }^{18}$ Figure $4(\mathrm{~b})$ shows the imaginary $\left(Z^{\prime \prime}\right)$ parts of impedance as a function of frequency at various temperatures. It reveals that $Z^{\prime \prime}$ first increases with the increase in both frequency as well as temperature and reaches a maximum $\left(Z_{\max }^{\prime \prime}\right)$ and then starts decreasing at higher frequencies. The appearance of peak in $Z^{\prime \prime}$ versus frequency at different temperatures suggests the presence of dielectric relaxation process in the material. It can also be seen that the $Z^{\prime \prime}$ peak of the plots are shifted toward higher frequencies side on increasing the temperature, which indicates the presence of temperature dependent relaxation process in the system. The broadening of the peak increases with increase in temperature, which suggests the spread of relaxation time. This may be due to the

Table 1. Values of bulk resistance $\left(R_{\mathrm{b}}\right)$ and bulk capacitance $\left(C_{\mathrm{b}}\right)$ of KNN ceramics at different temperatures.

\begin{tabular}{ccc}
\hline Temperature $\left({ }^{\circ} \mathrm{K}\right)$ & $R_{\mathrm{b}}(\Omega)$ & $\left(C_{\mathrm{b}}\right)(\mathrm{F})$ \\
\hline 703 & $1.393 \times 10^{6}$ & $6.178 \times 10^{-10}$ \\
713 & $5.704 \times 10^{5}$ & $4.989 \times 10^{-10}$ \\
723 & $2.627 \times 10^{5}$ & $4.563 \times 10^{-10}$ \\
733 & $1.469 \times 10^{5}$ & $4.115 \times 10^{-10}$ \\
743 & $8.746 \times 10^{4}$ & $3.527 \times 10^{-10}$ \\
753 & $5.543 \times 10^{4}$ & $2.923 \times 10^{-10}$ \\
763 & $4.540 \times 10^{4}$ & $2.584 \times 10^{-10}$ \\
773 & $3.409 \times 10^{4}$ & $2.137 \times 10^{-10}$ \\
\hline
\end{tabular}




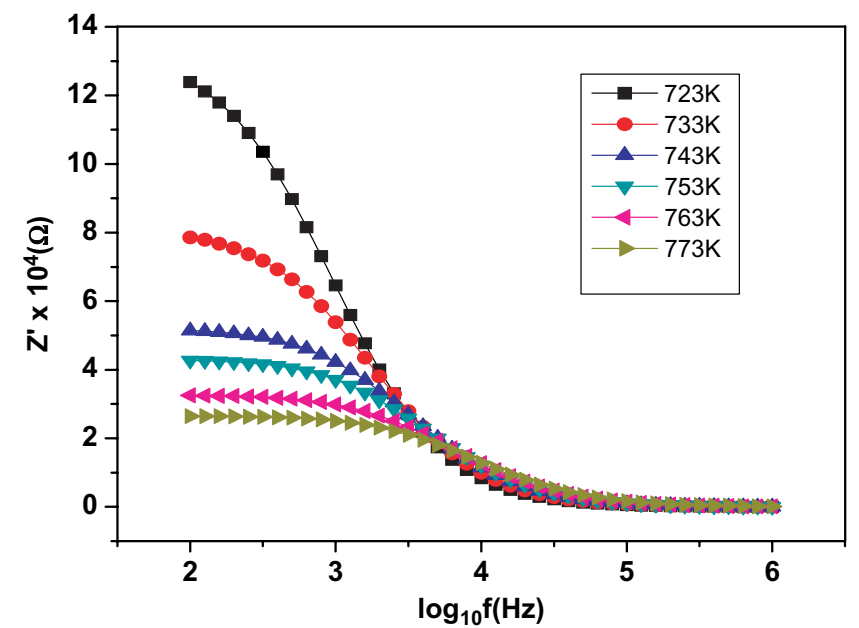

(a)

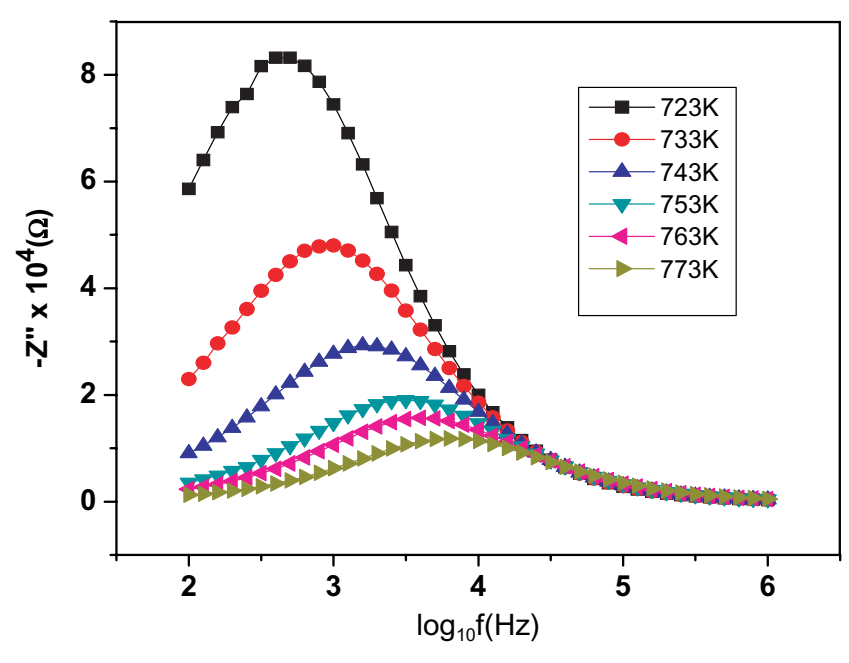

(b)

Fig. 4. (Color online) Frequency dependences of the real part (a) $Z^{\prime}$ and imaginary part $Z^{\prime \prime}$ (b) of impedance of KNN ceramics at various temperatures.

presence of defects at higher temperatures. The relaxation frequency can be obtained from the plots of $Z^{\prime \prime}$ versus frequency. The relaxation frequency obeys the Arrhenius relation given by

$$
\omega_{\max }=\omega_{0} \exp \left[-E_{\mathrm{a}} / k_{\mathrm{B}} T\right]
$$

where $\omega_{0}$ is pre-exponential factor and $k_{\mathrm{B}}$ is Boltzmann constant. The activation energy $E_{\mathrm{a}}$ is calculated from the slope of the graph of $\log \omega$ versus $1000 / T$, which is shown in Fig. 5. The calculated activation energy is found to be $\sim 1.46 \mathrm{eV}$.

Figure 6 shows frequency dependence of $\mathrm{AC}$ conductivity $\left(\sigma_{\mathrm{ac}}\right)$ at various temperatures. $\mathrm{AC}$

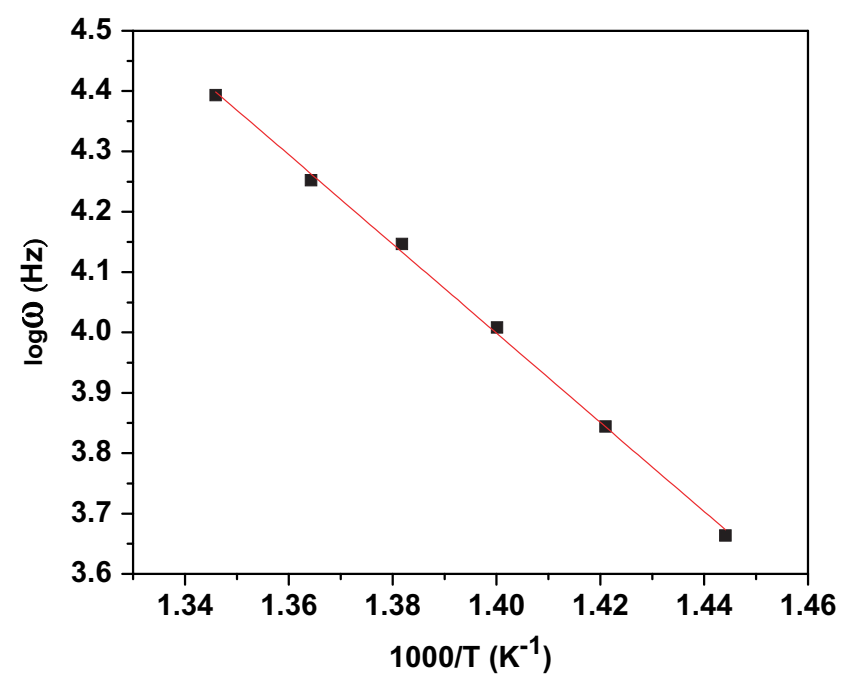

Fig. 5. Temperature dependence of the most probable relaxation frequency obtained from the frequency dependent plots of $Z^{\prime \prime}$ at different temperatures.

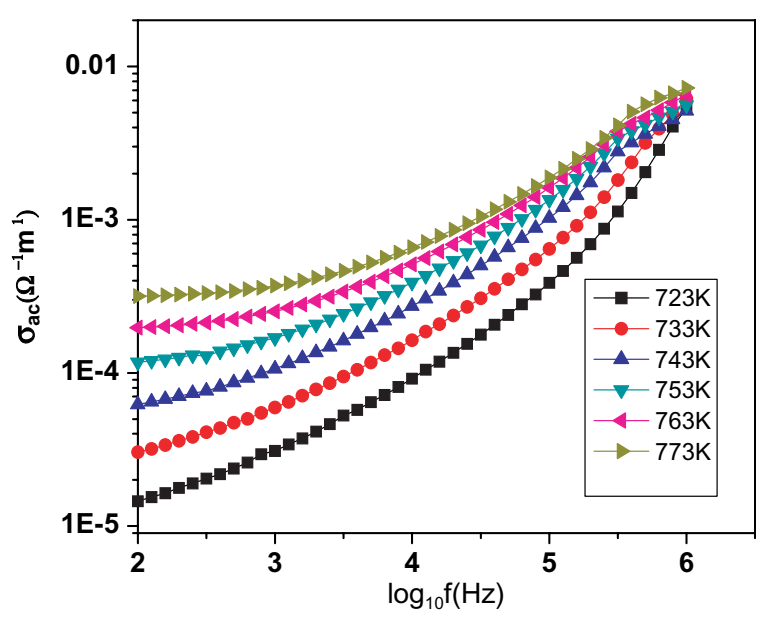

Fig. 6. (Color online) Frequency dependence of the AC conductivity of KNN ceramics.

conductivity was calculated using the empirical relation $\sigma_{\mathrm{ac}}=\omega \varepsilon_{\mathrm{r}} \varepsilon_{0} \tan \delta$, where $\varepsilon_{0}$ permittivity is in free space, and $\omega$ is angular frequency. The $\sigma_{\text {ac }}$ patterns show a frequency independent plateau in the low frequency region and exhibits dispersion at higher frequencies. This behavior satisfies the universal power law

$$
\sigma(\omega)=\sigma_{0}+A \omega^{n}
$$

where $\sigma_{0}$ is the DC conductivity (frequency independent plateau in the low frequency region), $A$ is the pre-exponential factor and $n$ is the fractional exponent, whose value lies in between 0 and 1 . It is observed that $\sigma_{\mathrm{ac}}$ increases with the increase in temperature which confirms the negative coefficient 


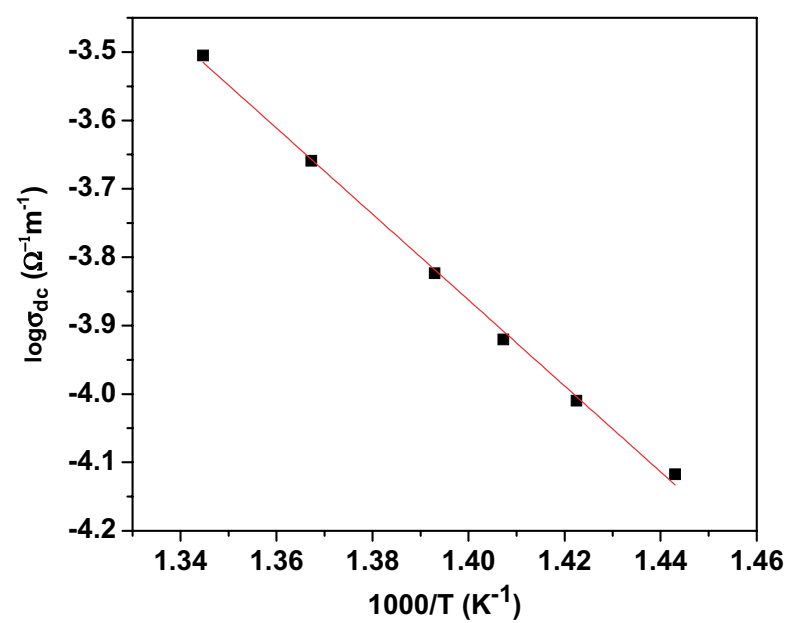

Fig. 7. Temperature dependence of the DC conductivity curve for KNN ceramics.

of resistance (NTCR) behavior. This behavior also suggests that the electrical conduction increases at the higher temperature which may be due to the increase in mobility of ions.

Figure 7 shows the variation of DC conductivity $\left(\sigma_{\mathrm{dc}}\right)$ with inverse of temperature. The nature of conductivity variation indicates an increase in conductivity with rise in temperature with a typical Arrhenius-type behavior. This suggests that the electrical conduction in the material is a thermally activated process which is governed by the relation: $\sigma_{\mathrm{dc}}=\sigma_{0} \exp \left[-E_{\mathrm{a}} / k_{\mathrm{B}} T\right]$. The activation energy, calculated from the slope of Fig. 7 , is $\sim 1.25 \mathrm{eV}$, which confirms that oxygen vacancies dominate the conduction process in the material in the paraelectric region.

Since, intrinsic cation vacancies are associated with oxygen vacancies. Therefore, in perovskite compounds in order to maintain the charge neutrality, oxygen vacancies are generated. In KNN ceramics, because of the volatile nature of the alkali elements $(\mathrm{Na} / \mathrm{K})$ during high temperature processing $\left(>800^{\circ} \mathrm{C}\right.$ ) cation vacancies are generated. Again as a result of this phenomenon, oxygen vacancies are created. It has been reported that oxygen vacancies have activation energies in the range $0.3-0.5 \mathrm{eV}$ for single-ionized oxygen vacancies and $0.6-1.2 \mathrm{eV}$ for doubly ionized oxygen vacancies, respectively. ${ }^{19-21}$ As a consequent, there is a probability of formations of double ionized oxygen vacancy with the release of single/two electrons due to heating of the KNN ceramics at high processing temperatures with the evaporation of more alkali elements. According to the
Kroger-Vink notation ${ }^{22}$ as given below.

$$
\begin{gathered}
\mathrm{Na}_{\mathrm{Na}} \rightarrow \mathrm{Na}+V_{\mathrm{Na}}^{\prime}+h^{\bullet} \\
\mathrm{K}_{\mathrm{K}} \rightarrow \mathrm{K}+V_{\mathrm{K}}^{\prime}+h^{\bullet} \\
\mathrm{O}_{\mathrm{O}} \rightarrow \frac{1}{2}\left(\mathrm{O}_{2}\right)+V_{\mathrm{O}}^{\bullet}+e^{\prime \prime} \\
\mathrm{O}_{\mathrm{O}} \rightarrow \frac{1}{2}\left(\mathrm{O}_{2}\right)+V_{\mathrm{O}}^{\bullet \bullet}+2 e^{\prime},
\end{gathered}
$$

the concentration of the sodium/potassium vacancies, $V_{\mathrm{Na}}^{\prime}$ or $V_{\mathrm{K}}^{\prime}$, would be double that of one or two oxygen vacancy(s), $V \bullet \bullet / V^{\bullet}{ }^{23}$ It is observed that the activation energy for relaxation frequency of charge carriers is more than that for conduction. This may be due to the fact that the activation energy for conduction is the sum of both the creation of charge carriers and migration of charge carriers over a long distance, whereas the activation energy for relaxation frequency of charge carriers is due to the migration of charge carriers and hopping of these charge carriers between the adjacent lattice sites. ${ }^{24}$

\section{Conclusions}

$\mathrm{K}_{0.5} \mathrm{Na}_{0.5} \mathrm{NbO}_{3}$ samples synthesized by a mixed oxide method at higher temperatures have been studied for its structural, dielectric and electrical properties. Complex impedance analysis confirmed the presence of a single semicircular arc in a wide temperature range. This indicates that the electrical properties of the material arise mainly due to the bulk effects. It also shows that bulk resistance $\left(R_{\mathrm{b}}\right)$ decreases with increase in temperature, which manifests NTCR behavior of the compound. The shifting of $Z^{\prime \prime}$ peak toward higher frequencies side on increasing the temperature indicates the presence of temperature dependent relaxation process in the system. The frequency dependent maxima of $Z^{\prime \prime}$ are found to obey Arrhenius law with an activation energy $\sim 1.46 \mathrm{eV}$, whereas the electrical conductivity also showed thermally activated behavior with an activation energy of $\sim 1.25 \mathrm{eV}$. The value of activation energy in both cases confirmed that the conduction process of KNN ceramic is due to doubly ionized oxygen vacancies.

\section{References}

1. B. Jaffe, W. R. Cook and H. Jaffe, Piezoelectric Ceramics (Academic Press, New York, 1971), pp. $221-224$. 
2. G. Haertling, J. Am. Ceram. Soc. 82, 797 (1999).

3. J. W. Waanders, Piezoelectric Ceramics - Properties and Applications (Philips Components, Eindhoven, 1991).

4. M. D. Maeder, D. Damjanovic and N. Setter, J. Electroceram. 13, 385 (2004).

5. L. Egerton and D. M. Dillon, J. Am. Ceram. Soc. 42, 438 (1959).

6. Y. Saito, H. Takao, T. Tani, T. Nonoyama, K. Takatori, T. Homma, T. Nagaya and M. Nakamura, Nature 432, 84 (2004).

7. G. Z. Zang, J. F. Wang, H. C. Chen, W. B. Su, C. M. Wang, P. Q. Ming, J. Du, L. M. Zheng, S. Zhang and T. R. Shrout, Appl. Phys. Lett. 88, 212908 (2006).

8. Y. Guo, K. Kakimoto and H. Ohsato, Appl. Phys. Lett. 85, 4121 (2004).

9. H. Takao, Y. Saito, Y. Aoki and K. Horibuchi, J. Am. Ceram. Soc. 89, 1951 (2006).

10. Y. Guo, K. Kakimoto and H. Ohsato, Solid State Commun. 129, 279 (2004).

11. S. Y. Chu, W. Water, Y. D. Juang, J. T. Liaw and S. B. Dai, Ferroelectrics 297, 11 (2003).

12. S. Lanfredi, L. Dessemond and A. Rodrigues, J. Am. Ceram. Soc. 86, 291 (2003).
13. I. Coondoo, A. K. Jha and S. K. Agarwal, Ceram. Int. 33, 41 (2007).

14. B. V. B. Saradhi, K. Srinivas, G. Prasad, S. V. Suryanarayana and T. Bhimasankaram, Mater. Sci. Engg. B 98, 10 (2003).

15. L. Liu, M. Wu, Y. Huang, Z. Yang, L. Fang and C. Hu, Mater. Chem. Phys. 126, 769 (2011).

16. P. Kumar and P. Palei, Ceram. Int. 36, 1725 (2010).

17. P. Kumar, S. Singh, O. P. Thakur, C. Prakash and T. C. Goel, Jpn. J. Appl. Phys. 43, 1501 (2004).

18. J. Suchanicz, Mater. Sci. Engg. B 55, 114 (1998).

19. C. Verdier, F. D. Morrison, D. C. Lupascu and J. F. Scott, J. Appl. Phys. 97, 024107 (2005).

20. R. Moos, W. Menesklou and K. H. Härdtl, Appl. Phys. A 61, 389 (1995).

21. R. Moos and K. H. Härdtl, J. Appl. Phys. 80, 393 (1996).

22. F. A. Kroger and H. Vink, J. Solid State Phys. 3, 307 (1956).

23. W. S. Lau and T. Han, Appl. Phys. Lett. 86, 152107 (2005).

24. A. Molak, E. Ksepko, I. Gruszka, A. Ratuszna, M. Paluch and Z. Ujma, Solid State Ion. 176, 1439 (2005). 